\title{
Analyzing Kerr Effect on WDM Optical Fiber Network with Fiber Bragg Grating
}

\author{
Abhineet Kaur ${ }^{1}$, Atul Mahajan ${ }^{2}$ \\ Student, Electronics \& Communication Department, \\ Amritsar College of Engineering and Technology, Amritsar, Punjab, India ${ }^{1}$ \\ Associate Professor, Electronics \& Communication Department, \\ Amritsar College of Engineering and Technology, Amritsar, Punjab, India ${ }^{2}$
}

\begin{abstract}
Since the event of the first-generation fiber communication systems in the early 80 's, the fiber communication technology has developed quickly to attain larger transmission capability and longer transmission distance, partially because of the increasing demand of network. The demand on the increasing system and network capability is expected to stay as additional bandwidth needed technologies like video conference and real-time image transmission emerge. Although global information management systems are capable of transmitting data at high data rates, they are still faced with many serious problems that reduce the performance of the optical transmission system as these Side effects. In our proposed work we found that parameters play a vital role in the development of the link, some of the values are interconnected with each other. E.g., power and frequency of CW laser are dependent on each other so we have to alter these two with utmost care. Specifications are changed under proper supervision so that not even a single one should be raised beyond the threshold value.
\end{abstract}

Keywords: Wavelength Division Multiplexing, Bragg Grating, Optical Fiber

\section{INTRODUCTION}

Since the event of the first-generation fiber communication systems in the early 80 's, the fiber communication technology has developed quickly to attain larger transmission capability and longer transmission distance, partially because of the increasing demand of network. The demand on the increasing system and network capability is expected to stay as additional bandwidth needed technologies like video conference and real-time image transmission emerge. To stay in step with the capability increasing demand, new devices and technologies are in great need. $[1,3]$ On the opposite hand, the systems should be rigorously studied and designed An optical fiber is a flexible, transparent, cylindrical, hair like structure made up of extruded glass or plastic slightly thicker than a human hair. Optical fiber is implemented where there is need of higher transmission data along with the requirement of larger bandwidth. The major advantage of optical fiber over traditional copper wires is that electromagnetic interference is absent in this transmission channel. Many measurements were made nowadays with fiber optic sensors. piece of equipment that adapts the optical rays into electronic signals. Both to resist the image, it detects the physical capacity of the light and converts it into the appearance of the instrument.[5]

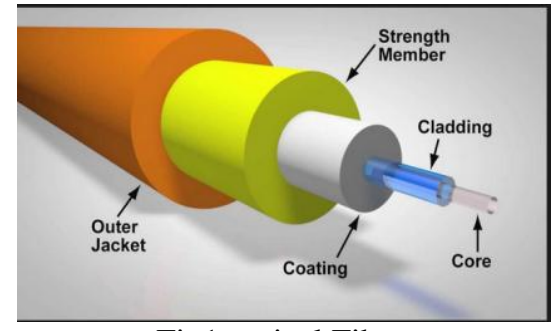

Fig1 optical Fiber

1 .Advantages of optical fiber: Fiber-optic systems have a large number of advantages over copper wire cables. Among the most important are the following:

- Because fiber-optic cables are both lighter and smaller in diameter than copper lines, they can be more easily produced and installed.

- Fiber-optic systems use significantly less energy than copper lines and are thus immune to many dangers associated with the electrical current used in copper lines. 
- Fiber-optic communication systems can be used to transmit more information than copper cables and are wellsuited for use with digital communications.

- When compared to copper cables, fiber-optic cables are again immune to electromagnetic interference and produce no interference when operating.

- Finally, fiber-optic lines are less expensive than copper cables, which can drastically reduce the cost of installing new lines or maintaining older ones.[4]

2 Cross Phase Modulation (XPM): High power optical amplifier and dispersion management change increased length of inter-amplifier spans in multi-channel high bit-rate transmission system. The bit-rate distance product is restricted by the combined effects of fiber dispersion and nonlinear effects as a result of the high launched power into the fiber. In the dispersion fiber, the combined effects of self-phase modulation (SPM), cross-phase modulation (XPM) and fourwave mixing (FWM) have an effect on the transmission performance. Phase-modulation due to XPM has important influence on a WDM systems because it affects channel over a continuous wavelength vary and is regenerate into distortion by residual dispersion.[6,2]

3 Four Wave Mixing (FWM): Four- wave mixing that is an undesirable feature in fiber propagation can be exploited to covert wavelength. Once a high powered optical signal is initiated a fiber, the linearity of the medium for optical response is lost. Four-wave mixing happens because of a third order linearity in silicon oxide fiber, referred to as optical Kerr impact.[7] Once 3 frequencies $\mathrm{f} 1, \mathrm{f} 2$ and $\mathrm{f} 3(\neq \mathrm{f} 1, \mathrm{f} 2)$ are initiated the fiber, it leads to a fourth wave of frequency $\mathrm{f}=\mathrm{f} 1+\mathrm{f} 2-\mathrm{f} 3$. The new wave is thought because the idler. Four-wave mixing causes undesirable impact in optical transmission once the probe wavelength is near the signal wavelength because the ensuing wave has the frequency of the sign as an example, in DWDM channels that are separated by a hundred rate, 3 wave of frequency GHZ, that is another channel within the WDM. So four-wave mixing would cause noise and cross talk.

Wavelength Division Multiplexing (WDM) is that the basic technology of optical networking. It's method for using a fiber (or optical device) to hold several separate and independent optical channels. The principle is similar to that used when we tune our receiver to 1of the many TV channels. Every channel is transmitted at a distinct radiofrequency and that we choose between them using a "tuner" that is just a electric circuit inside the receiving system. After all wavelength in the optical world is simply the manner we decide to see frequency and optical WDM is kind of similar to radio FDM.[8,11]

\section{RELATED WORK}

R.S Kaler (2012)In this paper [9], The 16 channel WDM at $10 \mathrm{~Gb} / \mathrm{s}$ are investigated for the varied optical amplifier and hybrid optical amplifiers and also the performance has been compared on the basis transmission distance and dispersion. The amplifier EDFA and SOA are investigated severally and further compared with hybrid optical amplifiers like RAMAN-EDFA and RAMAN-SOA. It is determined that hybrid optical amplifier RAMAN-EDFA provides the highest output power $(12.017$ and $12.088 \mathrm{dBm})$ and least bit error rate $(10-40$ and $9.08 \times 10-18)$ at the 100 kilometer for dispersion $2 \mathrm{ps} / \mathrm{nm} / \mathrm{km}$ and $4 \mathrm{ps} / \mathrm{nm} / \mathrm{km}$ respectively.

SachinChaugule, Ashish More (ICMEE 2010)In this paper [10], Wavelength Division Multiplexing (WDM) is the basic technology of optical networking. It is a way for using a fiber to hold several separate and independent optical channels. For long distance communication to realize error free reception use of repeaters is important repeaters are costly. Here we are using the combination of the optical amplifiers specifically Raman and EDFA. By using the mixture of these amplifiers with correct gain adjustment we will come through the bigger distance at that repeater may be placed that causes substantial advantage in reducing the no. of spans thereby reducing the total value for communication setup. Optical amplifiers the optical signal noise and distortion additionally amplified it.

A.K Srivastava (1999)In this paper [12], WDM is being introduced In Telecommunication network to handle the dramatic increase in traffic to many gigabits or terabits because of new services together with web. At such bit rate, various non-linear effects. In fiber limit the system performance. Link of a terrestrial WDM system should be, therefore designed to deal with these physical limitation. . This paper discusses the varied limitation expose by fiber once WDM transmission takes place. This paper also compares the performance of assorted styles of fibers suggested by ITU-T for WDM transmission and suggests various choices for dispersion management.

\section{PROPOSED METHOD}

The design Self-phase modulation, 2-channel Cross-phase modulation and 2-channel Four-wave mixing. I am also taking Fiber Bragg grating in the 2-channel FWM operated with basic optical communication system which consist of transmitter, transmission link and receiver to decline the noise. high data rate transmission at longer distance, with improved data rate and also with reduced power. Data transmission at Optical Fiber Network is studied with the BER analyze factor and opening of eye diagram. With help of this parameter we are able to know that our transmission is 
faithful or not. Without this parameter we can't transmit the data because we are not able to judge that transmission is occurred or not. By studying the value of BER, Q factor and opening of eye we get accurate output.

\section{SIMULATION RESULTS}

The Simulation Results After Applying Proposed Technique On Various Parameter Are Shown As

1.To study and design the system by using non-linear effects such as Self-phase modulation, Two channel Cross-phase modulation and Four-wave mixing using Fiber Bragg grating which consist of transmitter, transmission link and receiver.

The Simulation Results after Applying Proposed Technique on Various Parameter are shown as

\section{SPM Results}

1 Simulation result of SPM Q-factor when Bit rate is 5 Gbps in terms of Eye Diagram

\begin{tabular}{|l|l|l|l|}
\hline Bit Rate & Q-Factor & BER & Jitter \\
\hline 5 Gbps & 8.93084 & $1.96152 \mathrm{e}-019$ & 0.0021956 \\
\hline $10 \mathrm{Gbps}$ & 34.5393 & $1.15885 \mathrm{e}-261$ & 0.0022698 \\
\hline
\end{tabular}

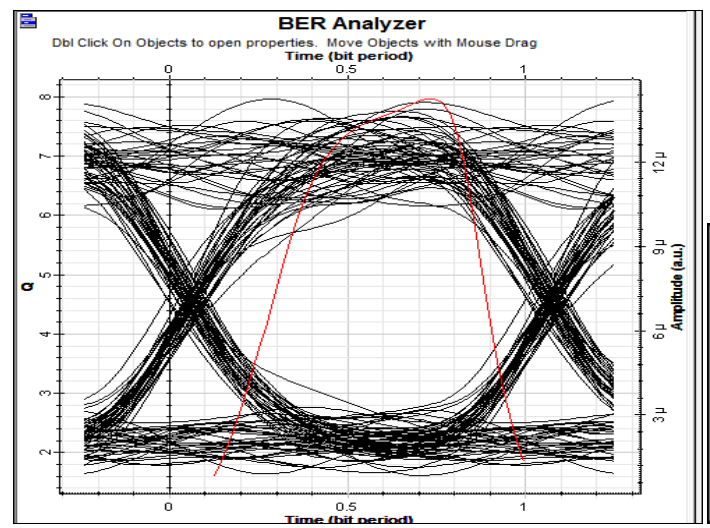

\begin{tabular}{||l|r|}
\hline \multicolumn{1}{||l|}{ Analysis } \\
\hline Max. Q Factor & 8.93084 \\
\hline Min. BER & $1.96152 \mathrm{e}-019$ \\
\hline Eye Height & $7.03241 \mathrm{e}-005$ \\
\hline Threshold & $5.0278 \mathrm{e}-005$ \\
\hline Decision Inst. & 0.546875 \\
\hline
\end{tabular}

Fig (2) Diagram of SPM Q-factor when Bit rate is 5 Gbps in terms of Eye Diagram

3. Simulation result of SPM Q-factor when Bit rate is $10 \mathrm{Gbps}$ in terms of Eye Diagram

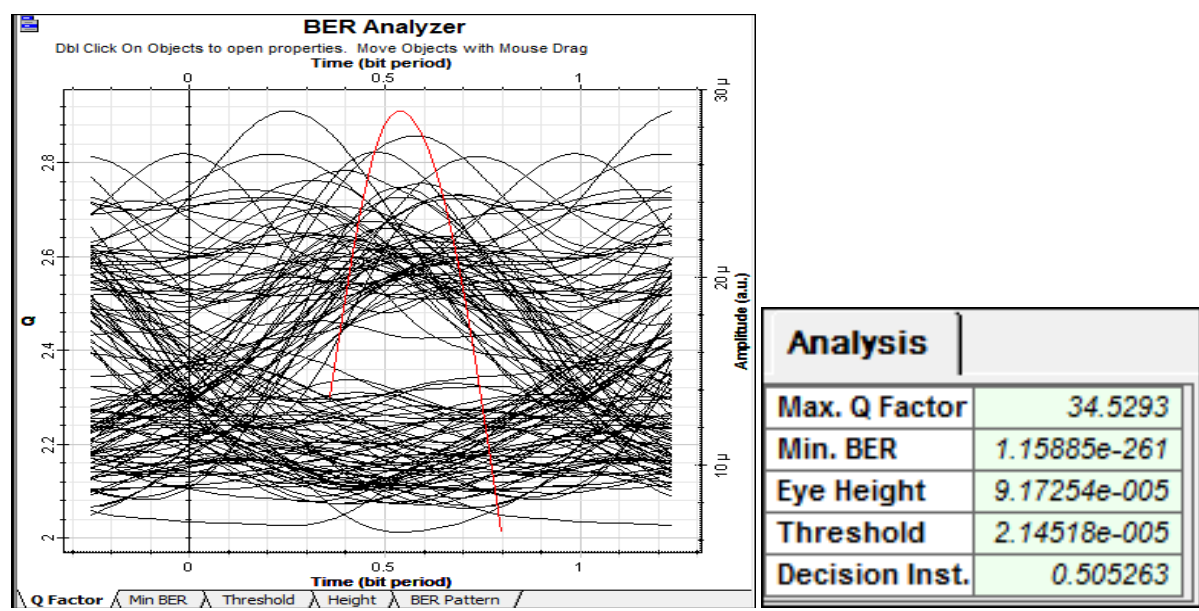

Fig (3) Diagram of SPM Q-factor when Bit rate is10 Gbps in terms of Eye Diagram

\section{XPM Results}

Table (4) for 2-channel XPM

\begin{tabular}{|l|l|l|l|}
\hline Bit Rate & Q-Factor & BER & Jitter \\
\hline 5 Gbps & 35.509 & $1.11852 \mathrm{e}-276$ & 0.0213563 \\
\hline $10 \mathrm{Gbps}$ & 4.977 & $2.42756 \mathrm{e}-007$ & 0.0156962 \\
\hline
\end{tabular}


1. Simulation result of 2-channel XPM Q-factor when Bit rate is 5 Gbps in terms of Eye Diagram

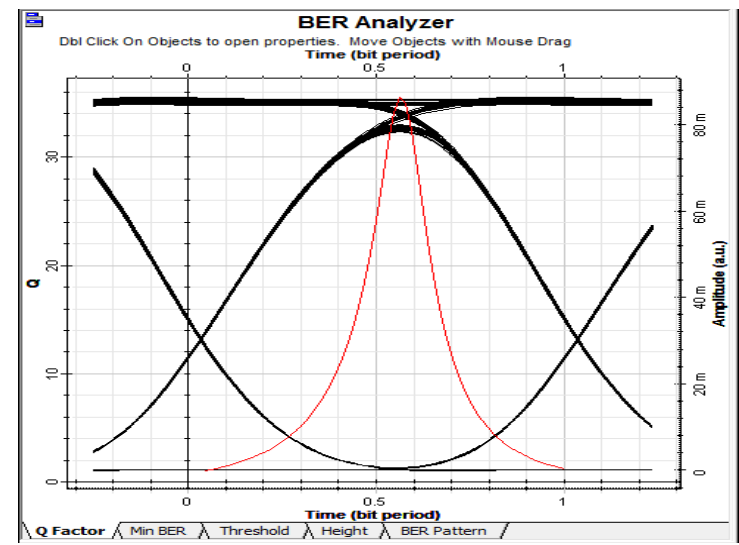

\begin{tabular}{||l|r||}
\hline \multicolumn{1}{||l|}{ Analysis } & \multicolumn{1}{|c|}{} \\
\hline Max. Q Factor & 35.5095 \\
\hline Min. BER & $1.11852 \mathrm{e}-276$ \\
\hline Eye Height & 0.0748933 \\
\hline Threshold & 0.00618702 \\
\hline Decision Inst. & 0.5625 \\
\hline
\end{tabular}

Fig (4) Diagram of 2-Channel XPM Q-factor when Bit rate is 5 Gbps in terms of Eye Diagram

2. Simulation result of 2-channel XPM Q-factor when Bit rate is $10 \mathrm{Gbps}$ in terms of Eye Diagram

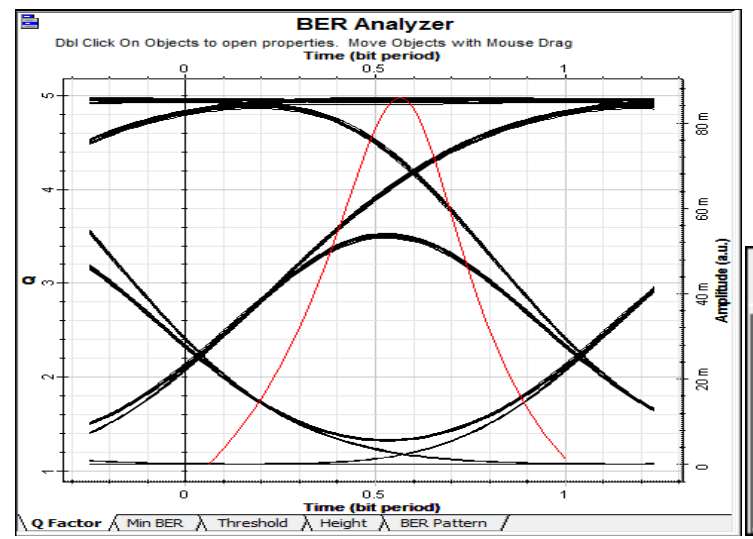

\begin{tabular}{||l|r|}
\hline \multicolumn{1}{|l|}{ Analysis } & \multicolumn{1}{|c|}{} \\
\hline Max. Q Factor & 4.97701 \\
\hline Min. BER & $2.42756 \mathrm{e}-007$ \\
\hline Eye Height & 0.0261964 \\
\hline Threshold & 0.0132366 \\
\hline Decision Inst. & 0.5625 \\
\hline
\end{tabular}

Fig (5) Diagram of 2-Channel XPM Q-factor when Bit rate is10 Gbps in terms of Eye Diagram

3. FWM Results

Table (5) for FWM

\begin{tabular}{|c|c|c|c|}
\hline Bit Rate & Q-Factor & BER & Jitter \\
\hline 5 Gbps & 11.4417 & $1.3701 \mathrm{e}-161$ & 0.0289523 \\
\hline $10 \mathrm{Gbps}$ & 11.1122 & $5.43167 \mathrm{e}-029$ & 0.0236952 \\
\hline
\end{tabular}

1. Simulation result of FWM Q-factor when Bit rate is 5Gbps in terms of Eye Diagram

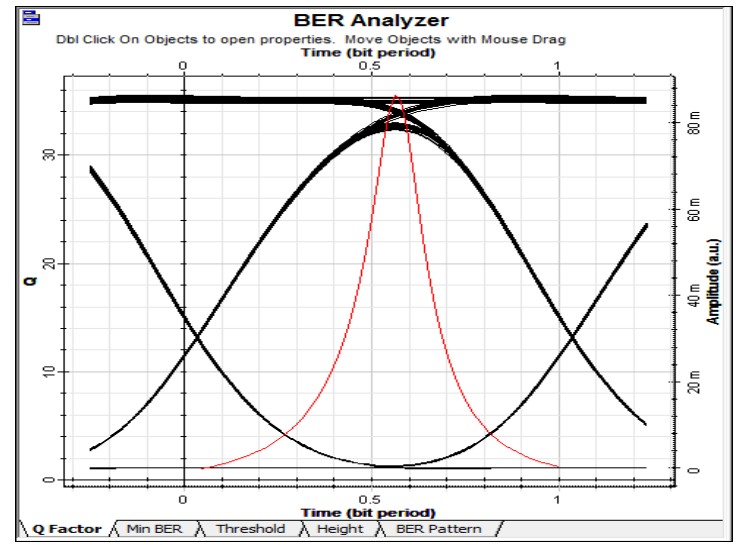

\begin{tabular}{|l|r|}
\hline Analysis & \multicolumn{2}{|l|}{} \\
\hline Max. Q Factor & 11.4417 \\
\hline Min. BER & $1.22737 e-030$ \\
\hline Eye Height & $7.59572 \mathrm{e}-005$ \\
\hline Threshold & $4.80232 \mathrm{e}-005$ \\
\hline Decision Inst. & 0.625 \\
\hline \hline
\end{tabular}

Fig (5) Diagram of FWM Q-factor when Bit rate is 5 Gbps in terms of Eye Diagram 
2. Simulation result of FWM Q-factor when Bit rate is $10 \mathrm{Gbps}$ in terms of Eye Diagram

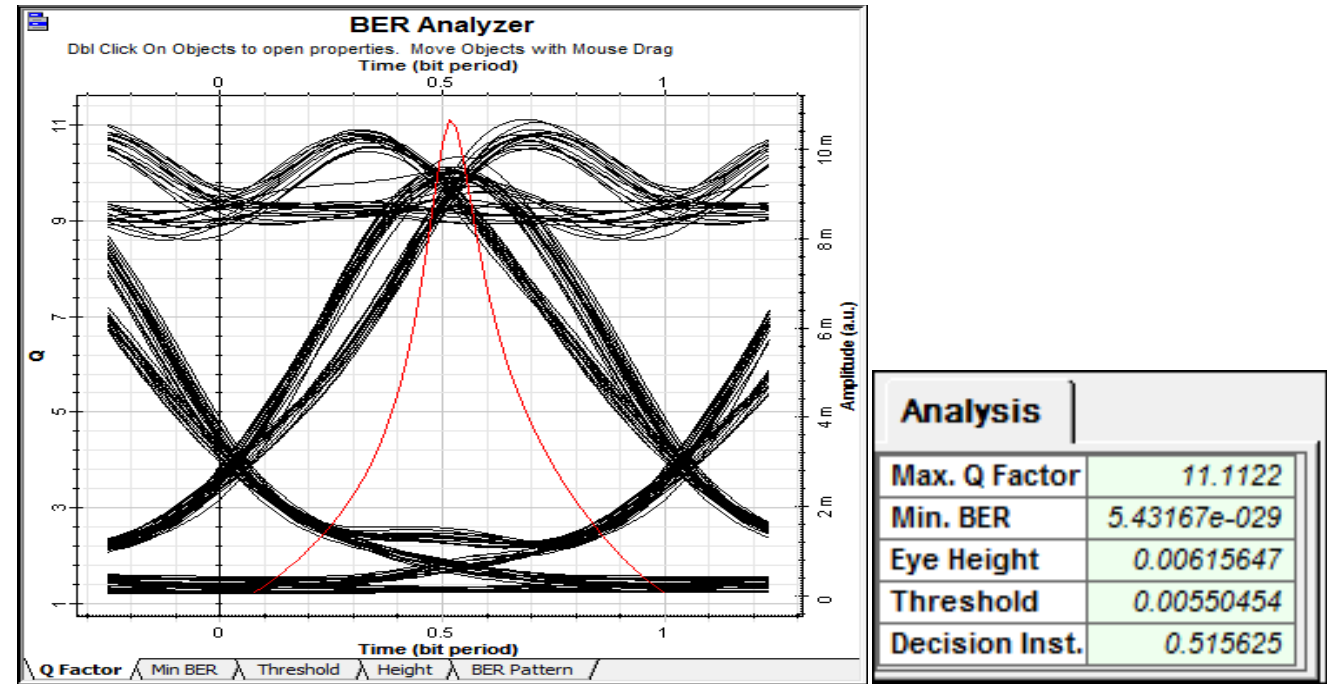

Fig (6) Diagram of FWM Q-factor when Bit rate is 10 Gbps in terms of Eye Diagram

\section{CONCLUSION}

The Kerr Effect is enhanced with Fiber Bragg Grating data rate and frequency have been studied. In this article, we had designed and simulated the nonlinear effects the overtime resources and the FWM on the WDM fiber optic network to negotiate huge data and reliable outcome . the Basic knowledge about each and every device/component, whether in the transmitting or in the channel or in the receiver section. Choice of better pulse generator among NRZ and RZ. Selection of a modulator which can perform multiple tasks at the same time thus saving/reducing the hardware and making the prototype more cost efficient and easy to operate. Data rate can be raised and transmission distance can be improved further with the implementation of FBG in non-linear effect which leads to better signal transmission and better synchronization in WDM networks.

\section{REFERENCES}

[1]. Inderpreet Kaur and Neena Gupta, "Comparative Analysis of Hybrid TDFA-EDFA and Hybrid EDFA-TDFA Configurations for 96 Channels DWDM System for S+C Bands", 2014 IEEE

[2]. Simranjit Singh and R. S. Kaler," Novel Optical Flat-Gain Hybrid Amplifier for Dense Wavelength Division Multiplexed System", IEEE Photonics Technology Letters, Vol.26, No. 2, January 15, 2014

[3]. M. Bhutani, and A. Gagneja, "Optical Transmission System Simulation for Analysis of Self Phase Modulation Non Linearity," International Journal of Scientific \& Engineering Research, vol. 4, no. 6, pp. 1707- 1713, June 2013.

[4]. Sugumaran S., VamsiNath Reddy P.," Design of WDM Optical Networks for the Analysis of Cross Phase Modulation and Self Phase Modulation”, Springer, CCIS 269, pp. 370-379, 2012.

[5]. Thele, H.J., Killey, R.I., Bayvel, P.: Transmission Limitations in Optical WDM Networks due to Cross Phase Modulation. The Institution of Electrical Engineers (1998)

[6]. Kikuchi, N., Sekine, K., Sasaki, S.: Analysis of Cross-Phase Modulation (XPM) Effect on WDM Transmission Performance. Electron. Lett. 33(8), 653-654 (1997)

[7]. S. Singh and R. S. Kaler,"Flat gain L-band Raman-EDFA hybrid optical amplifier for dense wavelength division multiplexed system," IEEE Photon. Technol. Lett., vol. 25, no. 3, pp. 250-252, Feb. 1, 2013.

[8]. Ronak R. Vashi, Arpan H. Desai and Amit H. Choksi," Modeling of Gain Flattening using EDFA-EYCDFA in Cascading Mode" International Journal of Emerging Trends \& Technology in Computer Science (IJETTCS), Volume 2, Issue 4, July - August 2013.

[9]. R.S. Kaler, "Simulation of $16 \times 10 \mathrm{~Gb} / \mathrm{s}$ WDM system based on optical amplifiers at different transmission distance and dispersion" Optik 123 , pp.1654-1658, 2012

[10]. Sachin Chaugule, Ashish More, "WDM and Optical Amplifier",2nd International Conference on Mechanical and Electronics Engineering (ICMEE 2010).

[11]. J. Toulouse, Optical nonlinearities in fibers: review, recent examples, and systems applications, J. Lightw. Technol. 23 (2005) $3625-3641$.

[12]. A.K. Srivastava, WDM transmission on existing optical fiber - anticipated problems and solutions, IETE Tech. Rev. 16 (1999) 335-339. 